\title{
Sustaining leprosy services in the changing context of health sector reform
}

\author{
A. T. GREEN \& K. JOCHEM \\ International Division, Nuffield Institute for Health, University of \\ Leeds, Leeds, UK
}

Accepted for publication 22 April 1998

\begin{abstract}
Summary National leprosy control programmes currently face a number of changes to the environment within which they operate. This paper examines the issues arising from these. It focuses, in particular, on those arising from changes in the structure of the health sector as a result of policies of health sector reform which are being considered or adopted in many developing countries. These include decentralization, financing strategies, greater role for the private and NGO sectors and the integration of vertical programmes. The paper is structured around a number of key steps in the development of a strategy for sustainability of appropriate leprosy services. These are the assessment of the epidemiological, social and health services context, development of programme objectives, planning of human and financial resources, development of the strategy, mapping the roles of potential actors, development of regulatory and incentive mechanism, action planning and managing change and, finally, re-evaluation of the programme objectives and service delivery organization. The paper stresses the importance of process in developing ownership of a strategy. It concludes with a set of key questions which it suggests need to be addressed by leprosy programme managers in the development of a proactive response to the changes.
\end{abstract}

\section{Introduction}

This paper looks at issues confronting national leprosy control programmes in the face of changes to the environment within which they operate. In particular, it focuses on those arising from changes in the structure of the health sector. It suggests key questions to be asked by each national programme as part of the development of a strategy for sustainability.

There are no universal answers to these questions. They need to be addressed in a contextspecific manner. This paper does not suggest responses to these questions or the context of

This paper is a revised version of a paper given by Andrew Green at the International Federation of Anti-Leprosy Associations (ILEP) Workshop on the Sustainability of Leprosy Services held in Amsterdam in September 1996. The comments of an anonymous referee are gratefully acknowledged.

Correspondence to: Mr A. Green, International Division, Nuffield Institute for Health, University of Leeds, 71-75 Clarendon Road, Leeds, LS2 9PL, UK 
any strategy. Many of the questions are not specific to leprosy, and could be asked of other disease control programmes, though the answers would differ.

Underpinning all this is the idea that the proactive and explicit development of a clear and well articulated strategy is critical to sustainability. 'Ad hocery' is unlikely to lead to sustainability. Three preliminary comments need to be made.

First, it is essential that the process be as broad as possible to develop a deep and wide sense of both understanding and ownership of the resultant strategy. This implies the involvement of agencies and individuals who may not traditionally be associated with leprosy. Such a process also requires clear leadership with an obvious role for government planning processes, which have a responsibility for setting the overall policy framework for the health sector, facilitated by the national leprosy programme. In some countries, these government processes may not be well developed. Especially in countries where leprosy services have historically been externally funded, it may be tempting to bypass the government planning process and develop strategies in a tighter (and narrower) fashion. It is, however, important for the long-term sustainability that these processes are fully involved. This may require the development of new facilitation and advocacy skills by leprosy programme managers.

Second, whilst many leprosy experts may feel that the answers to the key strategy questions are well-known, and even self-evident, it is still important to address them explicitly. This is partly as a result of the need to develop a broader constituency. However, the external environment is constantly changing, and truths that were held to be self-evident last year may be less certain this year.

Lastly, there is a need to see strategy development as dynamic and constantly evolving. The pursuit of sustainability is best served by the adoption of clear long-term goals coupled with short-term flexibility. This requires a cyclical process of monitoring and review, rather than the rigid pursuit of a set of targets and activities.

We look briefly at each set of the cyclical steps (summarized in Figure 1) required to develop a sustainable strategy.

\section{Assessing the epidemiological, social and health services context}

The first step involves an assessment of the current situation facing leprosy control in a particular country, together with any forseeable changes. The following sets out possible issues for country-based analysis in five broad (inter-related) areas:

- leprosy-related factors;

- health priorities;

- macro-economic factors;

- health sector composition and structure;

- attitudes towards leprosy and leprosy services.

\section{LEPROSY-RELATED FACTORS}

There are various factors concerning leprosy which are changing or which are uncertain. This paper does not focus on these, but they are mentioned for completeness and include the following. 


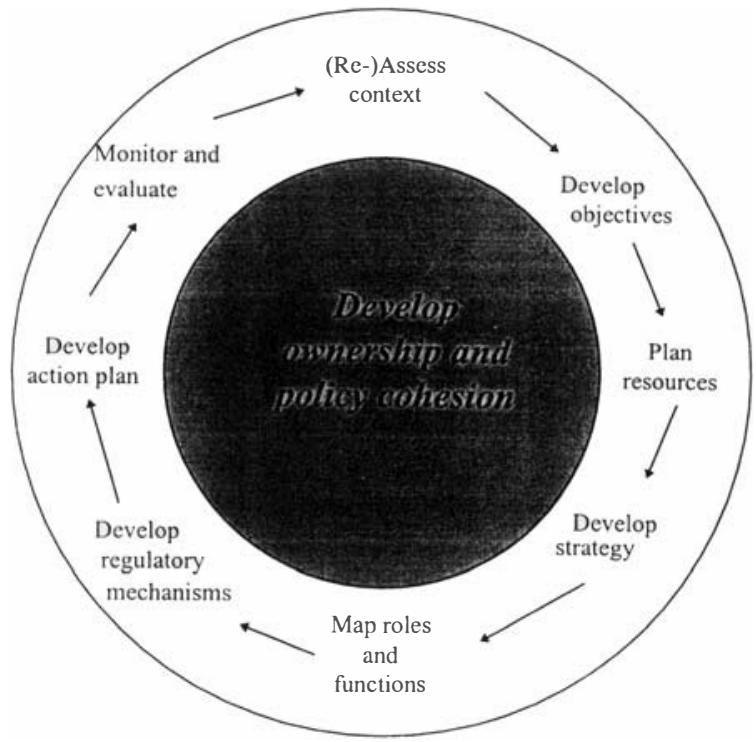

Figure 1. Steps in the development of a strategy.

The formal adoption by the World Health Assembly in 1991 of the goal to eliminate leprosy as a public health problem by the year 2000, and the endorsement of this goal at the first International Conference on the Elimination of Leprosy in Hanoi in1994, provides both opportunities and potential threats to leprosy programmes. ${ }^{4}$ While the World Health Assembly resolution has succeeded in putting leprosy elimination on national health agendas, the elimination goal has set up a new dynamic which potentially can divert programmes from the operational challenges of leprosy control. In particular, the choice of a definition of prevalence based on registered cases as a criterion of programme achievement has been criticized by some leprosy experts. ${ }^{1,5}$ While the wide use of MDT has certainly reduced the number of cases on treatment, the number of registered cases is not a reliable indicator of disease trends and is easily misused as a measure of disease burden, or as a guide to resource allocation and activity planning. This lack of consensus on the formulation of the goal has created tensions between the donor, technical and research bodies that need to be addressed.

Uncertainty as to the impact of increasing BCG coverage on reducing the future incidence of leprosy, ${ }^{2}$ the consequences of changes in the patterns of other diseases such as HIV, and the effects of non-medical factors such as poverty and urban crowding, ${ }^{3}$ all make less certain the short-term feasibility of an elimination goal, particularly at the local level, in high endemic countries.

A true reduction in incidence, if not a reflection of less intensive case-finding, will inevitably result in higher unit costs as the proportion of new cases in hard-to-reach subpopulations increases. This discordance between unit costs and progress toward the elimination target may lead to perverse pressures at the operational level if resource planning is too closely tied with numbers of registered cases, and not with the local requirements of improving early case detection and preventing and managing disabilities. The growing recognition of the importance of both functional and social rehabilitation outside long-stay 
institutions and leprosaria also has implications for the type and balance of services provided, which may imply additional short-term costs even as case numbers decrease.

\section{HEALTH PRIORITIES}

How any particular health problem is viewed has significant implications for the share of what, in most developing countries, are very limited resources available to the health sector. Whilst leprosy may never have been seen as a major priority within national governments, it has nonetheless been relatively successful in terms of fund-raising through the non-governmental organizations (NGO). One effect of the global strategy for elimination is that leprosy is likely to maintain, if not increase, its profile in the short term. However in the medium to long term, a combination of factors may lead to a reduction in its perceived importance. Falling prevalence and incidence will mean that leprosy is perceived more as a rare disease rather than as a public health problem. Furthermore, the growth in relative importance of other health problems, both infectious diseases (in particular HIV, STDs and TB) and non-communicable conditions, will have inevitable implications on the competition for resources.

There are also issues as to who sets priorities and on what criteria. There are various potentially competing approaches to priority setting which could have implications for the resultant priority given to particular health problems such as leprosy. These include the desire to include greater community participation in priority setting which was a critical component of the Primary Health Care strategy of Alma Ata. Recently, there has been increased emphasis on priority setting based on cost-effectiveness criteria, of which the most explicit is the Disability Adjusted Life Years (DALYs), advocated by the World Bank as a means of measuring the contribution of particular diseases to the overall burden of disease and hence the priority to be accorded to them. The values and assumptions that underpin such approaches may have important implications for leprosy and the resources allocated nationally to it. While the increase in the relative importance of disability versus death, and in the social and economic versus the medical consequences of disease implied by DALYs would logically value leprosy services, the actual effect may be the opposite in some countries if national priorities shift (perhaps under donor pressure) to a narrower range of basic health services deemed cost-effective.

The recent drive to decentralize decision-making and financing has implications for the potential balance between central and local priorities. Again, the impact on leprosy services may depend, for instance, on the mechanisms for financial decentralization, on what conditions may be set by central government to maintain certain services, and on the real opportunities for states and districts to redeploy health staff or reallocate financial resources.

\section{MACRO-ECONOMIC FACTORS}

Most developing countries have suffered a severe recession over the last decade, with little prospect of immediate change. This affects the context within which a leprosy strategy has to be developed.

First, and most obviously, the general recession has implications for the level of resources available. For countries which have adopted policies of structural adjustment, this exacerbates the already heavy pressures on the squeezed public sector. This, coupled with 
the potential medium-term decline in the perceived importance of leprosy, means that there is likely to be an eventual reduction in public sector leprosy resources.

Second, increasing poverty in sections of society arising both from recession and structural adjustment policies may make these more vulnerable to communicable diseases. Associated with poverty is an increased flow to the peri-urban populations and shanty towns. Continued growth in such densely populated areas may have implications for leprosy transmission. For South East Asian countries, which have managed to attain high economic growth rates, resource constraints are less likely, but the effects of urbanization may still be an issue.

\section{HEALTH SECTOR COMPOSITION AND STRUCTURE}

If the 1980s was the decade of Primary Health Care, the 1990s appears to be that of Health Sector Reform. International attention, highlighted, and in part led, by the World Bank 1993 Development Report, ${ }^{8}$ has focused on the structure of the health sector in an attempt to attain greater efficiency and responsiveness to public demand. Whilst there is no single Health Sector Reform package, there are common elements which countries are increasingly encouraged to consider. These can be summarized as:

- a conceptual split in the broad functions of health care policy development (including regulation and quality assurance), financing, and delivery of services, with the possibility of different agencies being responsible for different elements;

- a greater role for the non-State sector, including particularly NGOs and the private forprofit sector in the provision of care;

- decentralization of State services to allow greater autonomy and accountability to local areas;

- integration of vertical programmes linked in part to decentralization initiatives;

- the introduction of market approaches to management (performance-related pay and contracting) within the public sector;

- shifts in funding approaches with greater emphasis on individuals (user charges) rather than collective mechanisms (taxation) for financing health care.

The adoption of one or more of these elements of health sector reform is likely to have implications for leprosy programmes which need to be assessed as part of the development of a control strategy.

First, there is likely to be greater diversity of health care providers which may lead to a fragmentation of the sector. This, coupled with decentralization of the state sector, may lead to the danger of less policy cohesion. It may become harder to have an effective leprosy control policy.

Second, the potential reduction in the role of the public sector as a service provider may be mistakenly confused with a weakening of its role in the regulation and financing of health care. Both of these roles are, however, likely to be increasingly important. The increasing diversity of providers suggests the need for greater regulation and quality assurance. The particular nature of leprosy also suggests that all aspects of its care should continue to be collectively funded.

Third, and already mentioned, the processes of setting priorities may become more decentralized. This should not be interpreted as a reduction in the role of the Centre, but 
rather a change. There will continue to be a need for a strong central policy framework within which policy towards leprosy is set (this point is discussed further later).

\section{ATTITUDES TOWARDS LEPROSY AND LEPROSY SERVICES}

In the development of any strategy, an assessment of attitudes (often now called stakeholder analysis) towards the issue by different groups is essential, though often neglected. Insufficient attention to this critical area can lead to the development of elegant strategies which fail to be implemented due to a misunderstanding or failure to recognize and adjust to opposition to initiatives. Such analysis should identify all the key players in the process including NGOs, the private sector, other programmes of the health service and donors, as well as sections of the community, employers, industry and commerce including, in some countries, the pharmaceutical sector. One set of emerging key groups are leprosy patients' associations operating at both local and national levels.

\section{Developing programme objectives}

The second step in the development of the strategy is the setting of clear objectives. There are various issues related to this.

\section{THE ELIMINATION OBJECTIVE}

First, there is the country response to the WHO global strategy for elimination. At a basic level, there is the critical question as to whether it is broadly accepted. However, there are other issues, in particular the definition of the elimination goal in terms of the cases under treatment and how small-area denominator populations will be defined in translating the country targets into appropriate local targets. There are also questions as to whether measures of incidence will be used alongside prevalence measures. The development of decentralized priority setting as part of health sector reform may produce significant differences between health districts for the relative priority accorded to leprosy.

\section{BALANCE OF ACTIVITIES}

Second, the balance of activity between case detection, treatment and rehabilitation (both functional and social) needs to be reflected in the objectives. Routine programme monitoring should include the proportion of new patients with various disability grades, ${ }^{7}$ but as the number of cases decreases, monitoring should focus on delays in case detection and the activities related to the prevention of disabilities. ${ }^{6}$ A successful strategy must make explicit these questions of balance between different activities.

\section{WHAT IS TO BE SUSTAINED?}

At a broader level, there is the critical need to be clear as to precisely what the strategy is trying to sustain. There are at least three possible answers to this, with different implications for the subsequent strategy. The first level concerns the desire for a sustainable response to leprosy as part of broader health objectives, i.e. a concern that interest in leprosy does not 
diminish. Whilst this is entirely legitimate, particularly from a public health perspective, it is also important that a national strategy recognize that as the incidence of leprosy declines and it attains the status of a rare disease, communities may legitimately see it as of lower priority.

There may also be desire for the sustainability of dedicated leprosy activities either in the form of specific specialist services (such as diagnostic services or rehabilitation) or as vertical programmes. This objective, in either form, is hard to justify as a long-term aim, though it may be a necessary component under certain conditions for the attainment of wider health objectives.

Lastly, there may be a desire on the part of particular leprosy organizations to sustain their current roles. Leprosy organizations that have existed for some time may find it difficult to recognize when they have, through their own success, reached a situation where their role is either drastically changed or non-existent. It is however important, and the sign of a mature organization, that such organizations do not see their own existence as an objective in itself, but are prepared to reduce their activities or shift priorities. The involvement of leprosy NGOs in TB control or the use of leprosy personnel for TB work, which is happening or planned in several countries as the result of the new priority placed on TB, may be an appropriate response to shifting priorities.

\section{Planning human and financial resources}

One of the specific aspects of ensuring the sustainability of any service delivery concerns resources, including human resources. It is essential that any strategy developed is realistic in terms of its ability to raise the resources, both financial and physical, required to implement the strategy.

Whilst international NGOs (INGOs) may have been able to provide a significant proportion of such resources in the past, there is no guarantee that this will continue in the long term, particularly as the leprosy profile diminishes. The public sector's ability, in many countries, to devote resources to particular diseases is clearly limited by macro-economic constraints, the level of priority given (itself a function, among others, of public perceptions) and donor support. Current financing trends, away from tax-funded service provision towards user charges and social insurance, are likely to have implications also for leprosy, given its particular financing characteristics (chronic disease with a long treatment duration, relatively high drug and other treatment costs per individual, danger of defaulting and significant number of unemployed patients). Any strategy needs to examine carefully such issues and, where appropriate, develop arguments for continued public and INGO funding.

The planning and management of human resources is also a key issue. The support of leprosy staff towards changing strategies is likely to be critical to its success and yet a number, particularly those who have specialized in the field for many years, are likely to feel threatened by the changing environment. This may lead to resistance to the strategy. It is important that the development of a broad strategy towards future leprosy services pays particular attention to the implications for staff and any necessary redeployment and or retraining.

\section{Development of a strategy}

Once clear objectives concerning leprosy have been set, it is then necessary to develop a 
service strategy consistent with these objectives which takes account of the present and likely future external environment. Such a service strategy will specify the level (e.g. first-contact primary care services, general hospital, specialized facility) within the general health service and manner by which particular activities such as case detection or rehabilitation take place. At this stage, the focus should be on services rather than the agencies which will deliver them. The appropriate pattern of service delivery will be affected by a number of issues, including the following.

Reduced case numbers inevitably mean the need for different strategies to target hidden populations. It also raises the critical issue, faced by a number of countries already, as to whether to integrate with other vertical disease programmes or with general health services in order to capture economies of scale and reduce unit costs of service delivery. Such integration does not need to be wholesale and may, for example, be in the area of case detection with separate dedicated services remaining for rehabilitation. Ensuring that sufficient staff with specialized skills for disability management are available may require more flexible working arrangements, whereby certain staff are able to work in more than one health service jurisdiction. Integration may also be phased, with different elements being integrated at appropriate times as community resources for care and social rehabilitation are developed.

Lastly, it is also important to ensure that health sector reform policies of decentralization or integration are not confused with a reduction in the need for a strong technical resource for monitoring and technical policy guidelines. Experience in other fields suggests that decentralization in particular should not imply a weakening but rather a changing of the role of the centre. This may, for example, suggest a move away from managerial control as in vertical programme models, to one of technical leadership or quality assurance. Such a requirement may suggest a new role for former leprosy programme staff and possibly the development of new skills.

\section{Mapping the roles of potential actors in policy, financing and service provision}

The preceding step will result in a set of strategies for the delivery of services related to leprosy. From this will result a set of different functions which need to be assigned to different actors in the health care system. Table 1 sets out potential functional roles for the delivery of leprosy services and the likely main actors in the health care sector. The strategy needs to map, from such a set, the appropriate roles for different actors based on their strengths and weaknesses in each functional area. The changing nature of the health sector, and in particular increased emphasis arising from health sector reforms on the provision of services by non-State providers, has implications for this mapping exercise.

\section{Development of regulatory and incentive mechanisms}

Following the development of a clear service strategy and identification of roles for different actors, the strategy needs to develop a range of mechanisms to function as incentives and controls which will encourage or discourage the performance of these roles. If the strategy was designed to ensure that the growing diversity of private providers (likely under changing health structures) were able to recognize and refer early cases of leprosy, then appropriate incentives may need to be developed to ensure this. Similarly, if it was felt that an improved 
Table 1. Main functions and actors involved in leprosy services

Functions

Policy and regulation

- policy development

- technical guidelines

- quality assurance

- regulation

- monitoring

- surveillance

- advocacy

- research

\section{Service provision}

- BCG vaccination

- case detection

- laboratory services

- treatment (including MDT, disability prevention and palliation, management of reactions)

- specialist diagnostics

- functional rehabilitation (including surgery and prosthesis)

- social rehabilitation

- health education for patients \& families

- community awareness

- training for public \& private practitioners

- drug logistics

Financing

- government grants

- donations (including drugs \& equipment)

- local fund raising

- user charges
Potential actors

Government

- as policy maker

- as financer

- as regulator

- as health service provider at different levels

- specific MOH departments

- other government sectors

- local councils

NGOs

- local NGOs

- international NGOs

- volunteer organizations

- Leprosy Patients Associations

Private sector

- private hospitals

- licensed private practitioners

- drug companies \& distributors

- private pharmacists

- traditional practitioners

Labour and professional groups

- medical \& paramedical associations

- employers associations

- employee unions

Donors

- local donor

- international donors

Others

- technical agencies

- health training \& research institutes

- community and family

and broader information system was required as part of a monitoring process, then legislation to ensure compliance with this may be required.

\section{Action planning and managing change}

The implementation of a strategy requires a clear action plan. This needs to specify timebound activities related to the organization of services and raising of resources. In addition, however, and frequently neglected, is the need for an action plan that deals with the specific issues related to the management of organizational change in organizations such as NGOs whose role is likely to change as a result of the strategy. Managing change is important for at least two reasons. First, the shift in role, unless handled sensitively, may result in resistance to the strategy from key sections within the organization which are anxious, for a wide variety of reasons, to maintain the status quo. 
Table 2. Key questions in the development of a sustainable strategy for leprosy services

What is the current and future environment facing leprosy?

- how well are services currently meeting needs?

- how will the country respond to the WHO global strategy for elimination?

- is the balance of activities in the current services appropriate for now and the future?

What are the country's health priorities?

- how are they likely to change in the future?

- what does this mean for leprosy?

- who sets priorities and using what criteria?

What are the prospects for the country's economic situation?

- what are the implications for leprosy?

- what are the implications for general and leprosy-specific resources?

What changes to health sector structures are likely and how would this affect leprosy services?

- are the functional roles (policy, financing and provision) likely to be split?

- what is the role for the non-state sector (NGOs and private-for-profit)?

- what is the likely role for the state?

- is greater diversity of providers likely?

- is decentralization occurring or planned?

- is integration of vertical programmes occurring or planned?

- are changes in funding approaches planned?

- are there adequate regulatory and quality assurance mechanisms?

- is the monitoring and surveillance system appropriate for the changing sector?

What are the attitudes towards leprosy and leprosy services of key stakeholders?

- will they oppose, undermine or support change?

What are the objectives of the leprosy programme?

- what should be the balance of leprosy related activities?

- what are we aiming to sustain?

How will leprosy services be resourced in the future?

What should be the service strategy to meet the objectives?

- what activities should be carried out at what level in the service?

- is (phased) integration of specified elements of the leprosy service with other services appropriate?

- is there a mechanism and provision for adequate technical leadership?

- what should be the balance between case detection, treatment and rehabilitation?

Who are the main actors and what are their appropriate roles?

What regulatory and quality assurance mechanisms are required?

What should be the elements of the action plan for the management of change?

How will the plan be monitored and updated?

How will ownership of the strategy be developed?

Second, staff within such organizations represent an important reservoir of expertise and experience which can be tapped for the benefit of other areas. For example, the experience of leprosy organizations may be well directed towards the strengthening of TB programmes, or more general disability services.

\section{Re-evaluating programme objectives and service delivery organization}

The development of any plan for sustainability requires, as an essential element, the development of a continuous and explicit process of monitoring and review. This is critical to ensuring that progress towards the achievement of objectives is being made. As such, it 
becomes a tool for identifying problems early enough to rectify them. Such a process is also essential to predict any likely changes in the external environment and their potential effects on the strategy in order that any necessary changes to the strategy can be made. Sustainability will depend heavily on the ability to look forward, combined with a willingness to adapt flexibly to potential changes such as those likely to result from changes in the health sector.

\section{Achieving ownership and policy cohesion}

Lastly, and closely related to the previous element, is the need to ensure that there is broad ownership of the strategy both within the leprosy-specific organizations and, equally important, outside. Strong policy cohesion is particularly necessary in a situation where there is increasing sectoral fragmentation.

The development of broad ownership is most likely to be successful if involvement in the process of building a strategy starts from the beginning. Consultation amongst outside groups on polished strategies produced by a small internal group is often treated as tokenist, and rarely produces as deep a sense of identification as when there has been broad involvement in all stages of the overall strategic development.

It is important to recognize, however, that involvement in the development of a strategy will not necessarily lead to support for it. There may be groups or individuals who oppose the strategy either on genuine technical grounds or because they have a particular vested interest which may be challenged. Under such circumstances, an advocacy role to challenge such opposition may also be required as part of the strategy.

Government has a unique role to play in providing policy leadership, supported where appropriate by the specialist leprosy organizations. Mechanisms for ongoing co-ordination, once a strategy has been agreed, are also critical.

In conclusion, the preceding has set out a number of issues that face national leprosy programmes, with particular emphasis on the changing nature of the health sector as they enter the 21 st century. It has not attempted to provide prescriptions which must be context specific. Instead, it has provided a set of questions that need to be answered in the development of a strategy for the sustainability of appropriate leprosy services. These key questions are summarized in Table 2.

\section{References}

${ }^{1}$ Fine P. Reflections on the elimination of leprosy. Int J Lepr, 1996, 60: 71-80.

2 Karonga Prevention Trial Group. Randomised controlled trial of single BCG, repeated BCG, or combined BCG and killed Mycobacterium leprae vaccine for prevention of leprosy and tuberculosis in Malawi. Lancet, 1996; 348: 17-24.

3 Martelli CMT, Neto OLM, Andrade ALSS et al. Spatial patterns of leprosy in an urban area of central Brazil. Bull WHO, 1995; 73: 315-319.

${ }^{4}$ Noordeen S. Eliminating leprosy as a public health problem-is the optimism justified? World Health Forum, 1996; 17: 109-118.

5 Round Table Discussion. Eliminating leprosy a s a public health problem—is the optimism justified? World Health Forum, 1996; 17: 119-144.

6 Smith WC, Samson PD. Adapting leprosy programmes after MDT implementation. Tropical Doctor, 1997; 27: 193-194.

7 World Health Organisation. Guidelines for conducting a leprosy elimination programme review. International Conference on Elimination of Leprosy, Hanoi, 4-7 July 1994.

${ }^{8}$ World Bank. World Development Report 1993: Investing in Health. Oxford: Oxford University Press, 1993. 